\title{
MUNDIALIZACIÓN Y MEDIO AMBIENTE (LA OMC COMO AGENTE DEL DESARROLLO INSOSTENIBLE)
}

JOSÉ LUIS GORDILLO FERRÉ

Profesor Titular de Filosofía del Derecho

Universidad de Barcelona.

SUMARIO

0. INTRODUCCIÓN. 1. LA OMC Y EL MEDIO AMBIENTE. 2. COMERCIO Y VENTAJAS COMPARATIVAS. 3. ECONOMÍA Y RELACIONES DE PODER. 4. TRATO DISCRIMINATORIO Y PROTECCIÓN DEL MEDIO AMBIENTE. 5. EL IMPACTO AMBIENTAL DEL CRECIMIENTO ESTIMULADO POR EL COMERCIO "SIN INTERFERENCIAS”. 6. BIBLIOGRAFÍA.

\section{RESUMEN}

¿Es compatible el incremento de la producción, propiciado por la expansión planetaria del comercio, con el ideal del desarrollo sostenible, tal y como se afirma en el tratado fundacional de la Organización Mundial del Comercio? A nadie se le escapa que ésta es una gran pregunta cuya respuesta no se puede hacer en unas pocas páginas. Consciente de la magnitud de la cuestión, el autor de este artículo se limita a proponer unos cuantos apuntes críticos en torno a este asunto que, en cualquier caso, pueden servir para estimular la reflexión sobre un aspecto de la globalización que, a menudo, no es tenido en cuenta ni por sus defensores ni, lo que es peor, por algunos de sus detractores. 


\section{INTRODUCCIÓN}

Cualquier lector despierto, al leer el título de estas páginas, pensará que se encuentra ante un auténtico despropósito.

En efecto, si por un lado se alude a la mundialización y ésta se entiende como la "intensificación de las relaciones sociales en todo el mundo, por la que se enlazan lugares lejanos de tal manera que los acontecimientos locales están configurados por

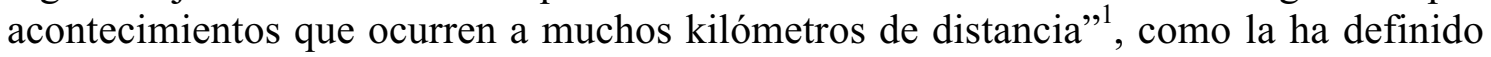
algún autor, y si, por otro, eso se pone en relación con el medio ambiente y este se entiende como equivalente a ecosfera ${ }^{2}$, entonces parece que tenemos la pretensión de abordar nada menos que la relación del todo social con el todo natural o, lo que es lo mismo, que queremos hablar del todo total.

Obviamente eso está más allá de nuestras posibilidades y de las de cualquier mente humana. Somos conscientes, como decían los clásicos, que no se puede hablar de todo a la vez y por alguna parte hay que empezar.

Nosotros empezaremos por aclarar que, de todos los aspectos que se pueden englobar bajo los rótulos "mundialización" o "globalización", a nosotros sólo nos va a interesar ahora su aspecto económico. Más en concreto, lo que nos interesa es la mundialización financiera y productiva.

En relación con el medio ambiente, de todos lo procesos que se han producido y se producen en la ecosfera, a nosotros sólo nos va a preocupar la crisis ecológica del presente, entendida como crisis de la relación de la humanidad con su hábitat provocada por la aproximación a (y en algunos supuestos sobrepasamiento de) los límites de la capacidad del planeta para sostener la vida humana.

Y al enlazar los dos asuntos, de lo que queremos hablar en realidad es del impacto en la crisis ecológica de un aspecto de la mundialización económica, a saber, de la expansión del comercio mundial impulsada por la OMC.

De todo lo que se puede discutir en relación con la mundialización neoliberal, vamos a centrar la exposición preferentemente en la OMC por dos motivos. En primer lugar, porque al hacerlo estaremos subrayando al mismo tiempo el lado político y coercitivo del citado proceso. Nada como el tratado fundacional de la O.M.C. para ilustrar que la mundialización que desemboca en el mercado mundial realmente existente, lejos de ser el resultado del azar, de una enigmática "naturaleza humana", de una todavía más misteriosa "necesidad económica" o del "libre" y "espontáneo" juego de la oferta y la demanda, es sobre todo una creación política ${ }^{3}$. En segundo lugar, porque en su tratado fundacional podemos encontrar elementos claros, explícitos e intersubjetivos de lo que se puede considerar como un proyecto embrionario de

\footnotetext{
${ }^{1}$ GIDDENS, A.: Consecuencias de la modernidad, Madrid, Alianza, 1993, págs. 67-68.

${ }^{2}$ En el sentido en que utiliza este concepto Barry Comonner, a saber, como el ecosistema de ecosistemas compuesto por la "fina envoltura de aire que rodea el planeta, el agua y el suelo y las plantas y animales que viven en él" producto de "los 5.000 millones de años de historia de la Tierra". Vid. COMMONER, B: En paz con el planeta, Crítica, Barcelona, 1992, págs. 11 y 15.

${ }^{3}$ Como ha dejado escrito el último Bourdieu. Ver: BOURDIEU, P.: Contrafuegos 2, Anagrama, Barcelona, 2001, pág. 104.
} 
gobierno económico mundial. En él, como veremos a continuación, retóricamente se incorpora una cierta preocupación por la protección de la ecosfera que constituye, asimismo, un buen exponente de lo que las élites occidentales piensan ahora sobre este asunto

\section{LA ORGANIZACIÓN MUNDIAL DEL COMERCIO Y EL MEDIO AMBIENTE}

Aunque su origen más remoto tal vez haya que buscarlo en la colonización de América y de otras partes del mundo, el proceso de mundialización en el que estamos inmersos recibió un claro impulso con las políticas neoliberales que comenzaron a aplicar Thatcher y Reagan a finales de los años setenta y principios de los ochenta, tanto en el plano interno como en el internacional. Se trata de un proceso alargado en el tiempo que acaba cristalizando, entre otras cosas, en la fundación de la Organización Mundial del Comercio en $1995^{4}$.

\section{El principio general en el que se inspira la O.M.C se puede resumir en la siguiente frase: "Los gobiernos deben interferir en el comercio lo menos posible".}

Por otro lado, en el preámbulo del acuerdo se dice que:

“... las partes reconocen que su relación en la esfera de la actividad comercial y económica deben tender a elevar los niveles de vida, a lograr el pleno empleo y a un aumento de los ingresos reales y la demanda efectiva y a acrecentar la producción y el comercio de bienes y servicios, permitiendo al mismo tiempo la utilización óptima de los recursos mundiales de conformidad con el objetivo de un desarrollo sostenible y procurando proteger y preservar el medio ambiente e incrementar los medios para hacerlo, de manera compatible con sus respectivas necesidades e intereses según los diferentes niveles de desarrollo económico."

La OMC, por tanto, parte de la presunción de que es compatible el incremento de la producción de bienes y servicios, gracias al desarrollo del comercio mundial, con la protección del medio ambiente.

Sobre el papel, la alusión al medio ambiente y al desarrollo sostenible supone un cambio notable respecto a lo que fue en el pasado la doctrina oficial sobre este tema. Hasta bien entrados los años setenta, ni siquiera se planteaba su supuesta compatibilidad, sino que se afirmaba, sin más, que todo desarrollo económico tenía unos costes medioambientales inevitables que había que aceptar porque, en cualquier caso, no constituían una grave amenaza para el conjunto de la humanidad. En esa línea, el presidente norteamericano Ronald Reagan llegó a declarar, en 1980, que respecto a la

4 La OMC es algo así como un "marco institucional común” que acoge en su seno a más de dos docenas de acuerdos de naturaleza variada. Para citar sólo los más relevantes: a) Acuerdo General sobre aranceles y Comercio; b) Acuerdo General sobre el Comercio de Servicios; c)Acuerdo sobre la Agricultura; d) Acuerdos sobre los aspectos de los Derechos de Propiedad Intelectual relacionados con el Comercio; e) Acuerdo sobre Medidas de Inversión relacionadas con el Comercio; f) Acuerdo sobre Obstáculos Técnicos al comercio; g) Acuerdo sobre Medidas Sanitarias y Fitosanitarias; h) Entendimiento relativo a normas y procedimientos por los que se rige el arreglo de controversias mediante el Órgano de Solución de las Diferencias. La OMC está dirigida por una secretaría con sede en Ginebra y cuenta con 140 estados miembros. 
responsabilidad humana en el desencadenamiento de la crisis ecológica no había que exagerar, ya que el principal agente contaminador del aire era "la vegetación". Unos periodistas presentes en la rueda de prensa bautizaron tan documentada afirmación como la "teoría de los árboles asesinos".

Hoy en día ningún dirigente político se atreve a repetir semejante sandez, y todavía menos el presidente del Estado más poderoso e influyente del planeta. Desde, como mínimo, mediados de los años ochenta, mostrar alguna clase de sensibilidad ecológica forma parte de la "corrección política" que ningún político profesional se atreve a desafiar, al menos en público.

Volviendo a lo que nos ocupa, el mencionado párrafo del tratado de la OMC plantea una gran interrogante: ¿es realmente compatible el incremento de la producción, propiciado por la expansión planetaria del comercio, con la protección del medio ambiente y el ideal del desarrollo sostenible?

A nadie se le escapa que una contestación rigurosa a esta gran pregunta no se puede hacer ni en unas pocas líneas ni en unas pocas páginas. Aquí vamos a limitarnos a exponer un par o tres de apuntes críticos con el objetivo de incitar a pensar y a discutir sobre una cuestión que es, con toda seguridad, de las más importantes de nuestra época.

\section{COMERCIO Y VENTAJAS COMPARATIVAS}

La creencia de que la produción se puede incrementar gracias al comercio, se apoya en la llamada "Teoría de las ventajas comparativas", cuya primera formulación se atribuye a David Ricardo (1772-1823) ${ }^{6}$.

Según esta teoría, cada país debe concentrarse en fabricar o en comercializar las mercancías que tengan el coste comparativo más bajo posible. Con ello se espera obtener grandes ingresos vendiéndolas en el mercado internacional. Con esos ingresos, a continuación, se obtiene una acumulación de capital que posibilita la elevación del nivel de vida de las poblaciones. La especialización, además, propicia una división internacional del trabajo que comporta un abaratamiento general de la producción hasta llegar a una situación planetaria de equilibrio económico cuasi perfecto.

Pero como ha señalado entre nosotros Roberto Bermejo $^{7}$, para que la teoría de las ventajas comparativas se pueda aplicar con garantías de éxito deberían darse las siguientes premisas:

\footnotetext{
${ }^{5}$ Vid. GREEN, M y McCOLL: Ronald Reagan, el rey del error, Fundamentos, Madrid, 1986, pág. 103. Asimismo, el secretario de interior con Reagan, James Watt, explicaba por entonces que la polución del planeta no debía ser una preocupación, pues "la vuelta del Señor está cerca" (Le Monde Diplomatique, ed. española, septiembre 2002, pág. 2). Todo eso ocurría después de la publicación del informe de prospectiva Global 2.000, encargado por el antiguo presidente James Carter, elaborado por todos los departamentos de la administración norteamericana y cuyas conclusiones sobre la crisis ecológica eran claras y explícitas. Esto puede dar una idea del grado de cinismo que expresan dichas declaraciones y de la irresponsabilidad criminal de quien las hizo.

${ }^{6}$ Vid. BERMEJO, Roberto: Libre comercio y equilibrio ecológico, Bakeaz, Bilbao, 1996, págs. 69 y ${ }^{\text {SS.. }}{ }^{7}$ Idem, pág. 71 .
} 
Los precios se establecen en función del libre juego de la oferta y la demanda.

No existen o son irrelevantes los costes de transporte.

No hay impacto ambiental.

Hay demanda suficiente para los productos intercambiados.

El coste del trabajo es uniforme.

Todos los países ganan con el comercio porque todos tienen ventajas comparativas.

Existe una misma dinámica de desarrollo tecnológico para cada producto.

Existe una misma dinámica de desarrollo de los mercados

Existe una inmovilidad internacional de determinados factores (para Keynes, por cierto, uno de ellos era el capital financiero).

La mera enunciación de todos estos factores ya permite intuir el carácter quimérico de la susodicha "Teoría". En el mundo real, como muy bien explica Bermejo, hay numerosos países que no pueden ofrecer ninguna ventaja comparativa; se produce fenómenos de saturación de determinados productos en los mercados; no todos los países tienen el mismo nivel de desarrollo tecnológico; el coste del trabajo no es uniforme, etc, etc. Sin embargo, no voy a comentar y criticar todas y cada una de estas premisas. Para lo que aquí interesa, es suficiente con centrarnos en las tres primeras.

\section{ECONOMÍA Y RELACIONES DE PODER}

Como asimismo señala Bermejo con acierto, nunca en ninguna parte el precio de los productos se ha establecido a partir del libre juego de la oferta y la demanda, sino que ha sido siempre el resultado de una "compleja interacción entre costes de producción, oferta, demanda y relaciones de poder"».

La época que nos ha tocado vivir ofrece inumerables ejemplos de que, en efecto, las relaciones de poder intervienen ( iy de qué manera!) en la fijación de precios y en muchos otros aspectos de la actividad económica.

Según un informe de la UNCTAD del año $2.000^{9}$, en el mundo existen alrededor de 60.000 empresas transnacionales, es decir, que tienen plantas o instalaciones en varios países a la vez. De ellas, las 200 primeras controlan por sí solas el $25 \%$ de todo lo que se produce en el mundo. Muchas de las empresas restantes, por la vía de la subcontratación, mantienen relaciones clientelares con ellas.

Las grandes empresas transnacionales controlan, en régimen de oligopolio, sectores enteros de la actividad económica planetaria. Así, por ejemplo, entre 3 y 6 empresas controlan entre el $70 \%$ y el $90 \%$ del sector alimentario, el de las materias primas agrícolas, el de extracción de minerales y el de la comercialización de los combustibles fósiles. Alrededor de 5 empresas controlan más del 50\% del sector del automóvil, del

\footnotetext{
${ }^{8}$ Ibidem.

${ }^{9}$ Se trata del World Investment Report 2000, UNCTAD, New York y Ginebra, 2001, pág. 71.
} 
transporte aéreo, de la industria aeroespacial, del sector eléctrico, de la electrónica y de la industria siderúrgica ${ }^{10}$.

En esos sectores hablar de "libre juego de la oferta y la demanda" es un sarcasmo. Los precios de los productos son el resultado más de acuerdos entre empresas que no de la competencia entre ellas ${ }^{11}$ Cualquier análisis y valoración de la globalización que no tenga en cuenta lo anterior no se puede considerar ni serio ni riguroso.

Según datos de Naciones Unidas y del Banco Mundial, una tercera parte del comercio mundial es intercambio entre filiales de la misma empresa transnacional (IBM-España con IBM-Italia, etc.). Otra tercera parte corresponde a transacciones entre las 500 empresas transnacionales más poderosas (Sony con IBM o Exxon con BP, etc.). Sólo la tercera parte restante se corresponde con transacciones entre empresas nacionales $^{12}$.

A partir de estos datos no resulta difícil determinar quienes están más interesados en la expansión del comercio mundial y en que éste se desarrolle de acuerdo con las normas de la actual OMC. Su misma existencia se debe, según testimonio de David Hartridge, actual director de su división de servicios, a la “ ... enorme presión ejercida por el sector financiero norteamericano, especialmente por parte de compañías como American Express y Citicorp". Sin ella, añade Hartridge, "no habría existido acuerdo alguno sobre el comercio de servicios y, por lo tanto, quizás tampoco la Ronda de Uruguay ni la $\mathrm{OMC}^{, 13}$.

Son esas megacorporaciones, pues, las que están interesadas en que los gobiernos "interfieran lo menos posible" en el comercio mundial.

Esa inmensa acumulación de poder económico es inexplicable si no se toma en consideración, paradójicamente, la inmensa capacidad de influencia de los Estados más poderosos en la configuración de orden económico internacional. Más en concreto: lo que allanó el camino para la expansión de la mundialización neoliberal fueron los famosos planes de ajuste, impuestos a raíz de la crisis de la deuda mexicana, a decenas de países pobres por los Estados agrupados en el G-7 y a través de organismos como el Fondo Monetario Internacional y el Banco Mundial. Dichos planes, inspirados en el más crudo neoliberalismo, siempre han exigido la apertura de las fronteras a los productos y a las inversiones extranjeras. Por esta vía se facilitó la penetración de las empresas transnacionales.

Todavía hoy, los gobiernos de los países ricos del G-7 son los que deciden las directrices básicas de la política económica de los Estados endeudados. La forma de gestionar su inmensa deuda externa sólo puede verse, a estas alturas, como un mecanismo de neocolonialismo económico.

\footnotetext{
${ }^{10}$ Vid. BERMEJO, R., op. cit., pág. 91.

${ }^{11} \mathrm{Y}$ cuando se llevan a cabo privatizaciones en estos sectores, lo que se está facilitando no es un aumento de la competencia, como dicen los propagandistas neoliberales, sino las absorciones y megafusiones entre gigantes empresariales.

${ }_{12}$ Datos citados por GEORGE, Susan: Pongamos a la OMC en su sitio, Icaria, Barcelona, 2002, pag. 12 y también BERMEJO, R., op. cit., pág. 77.

${ }^{13}$ Cit. en GEORGE, S., op.cit., pág. 13.
} 
Gracias a él, las grandes empresas deciden ahora la ubicación geográfica de sus plantas industriales en función del mayor o menor coste de la mano de obra y de la existencia o no de legislación medioambiental. La mundialización neoliberal en curso, en sí misma, permite que las empresas burlen con mucha facilidad los controles de los Estados.

Cabe apuntar que a dichas empresas se les quiere conceder mucho protagonismo en el futuro "Acuerdo General sobre el Comercio de Servicios", todavía en fase de discusión. La principal propuesta objeto de debate versa sobre la privatización de todos los servicios públicos de los que los Estados obtengan alguna ganancia comercial o que los ofrezcan en competencia con empresas privadas. Eso incluye los "servicios medioambientales". La propuesta negociadora de la UE incluye, por ejemplo, la privatización de servicios como "tratamiento de residuos", "recolección, purificación y distribución del agua" o la "prevención y solución de daños ambientales" ${ }^{14}$. De lo que hay que deducir que los defensores de la OMC creen posible alcanzar un desarrollo sostenible a través de la negociación privada entre empresas o mediante los denominados "códigos de conducta" para la autorregulación de su actividad ${ }^{15}$. Sólo los creyentes más fanáticos en el "milenarismo neoliberal" pueden creerse a pies juntillas este auténtico cuento de la lechera.

\section{TRATO DISCRIMINATORIO Y PROTECCIÓN DEL MEDIO AMBIENTE}

Es cierto, de todas formas, que según el tratado fundacional de la OMC los Estados pueden seguir aplicando medidas para la protección del medio ambiente. Pero deben hacerlo siempre y cuando éstas no se utilicen a modo de barreras proteccionistas.

Así, en el preámbulo del tratado fundacional también se dice que:

"Deseosas de contribuir al logro de estos objetivos mediante la celebración de acuerdos encaminados a obtener, sobre la base de la reciprocidad y de mutuas ventajas, la reducción sustancial de los aranceles aduaneros y de los demás obstáculos al comercio, así como la eliminación del trato discriminatorio en las relaciones comerciales internacionales."

Por tanto, políticas medioambientales sí, pero siempre que no constituyan una barrera proteccionista que comporte un trato discriminatorio a los productos extranjeros, dado que el objetivo primordial del tratado es favorecer el comercio. Como dice Susan George, la consigna de los librecambistas de la OMC es "comercio ubër alles", el comercio por encima de todo.

¿Cuándo una política medioambiental de un Estado se puede calificar de medida discriminatoria para un producto extranjero? En la práctica eso depende de la interpretación discrecional que haga la autoridad encargada de resolver las denuncias

\footnotetext{
${ }^{14}$ Vid. ARMBORST, Stefan: "Liberalizar y comercializar todo bajo el sol" en El Ecologista, n”28, especial "Globalización y medio ambiente", nov. 2001, pág. 9-13; ver también: GEORGE, S., op. cit., pág. 49 y ss.

${ }^{15}$ Para una crítica de esta quimera, que al mismo tiempo lo es de la teoría jurídica de la autopóiesis, ver: OST, François: “ La auto-organisation écologique des entreprises: un jeu sans conflits et sans règles?” en la Rev. intern. ét. jur.(R.I.E.J.),1992/28, págs. 147-178.
} 
que presentan los Estados por incumplimiento de los tratados de la OMC. Esa autoridad es el Órgano de Solución de las Diferencias (OSD), compuesto por una serie de "expertos" (en su mayoría especialistas en derecho mercantil o en política comercial) que primero aconseja, si así lo estima oportuno, que se derogue esa medida que se considera discriminatoria, y si el Estado en cuestión no lo hace, entonces autoriza la adopción de las "represalias cruzadas", esto es, que el Estado que se sienta objeto de la discriminación puede, a su vez, impedir que entren en el mercado nacional productos fabricados en el Estado al que se considera culpable de discriminación comercial.

En los siete años de funcionamiento de la OMC, el OSD ha conseguido que diversos estados deroguen diversas leyes de protección del medio ambiente por este motivo $^{16}$.

Hasta la fecha, la ponderación que ha hecho el Órgano de Solución de las Diferencias entre políticas medioambientales y libre comercio ha ido claramente en detrimento de las primeras. Tanto es así que, desde las organizaciones ecologistas, se ha calificado de "contrarreforma ambiental a escala planetaria" ${ }^{17}$ el proceso impulsado por la OMC.

A lo que se debe añadir lo que se prevé en los proyectos en curso que se encuentran todavía en fase de negociación. Así, por ejemplo, en la reunión ministerial celebrada en Doha, del 9 al 14 de noviembre de 2001, se decidió llevar a cabo negociaciones sobre la relación entre los tratados de la OMC y los tratados multilaterales sobre medio ambiente. El primer acuerdo que se adoptó fue que las conclusiones a las que se llegara sólo afectarían a los estados firmantes de los tratados medioambientales. Ésto, como dice Susan George ${ }^{18}$, es un incentivo para que todos los estados sigan el ejemplo de EE.UU. en relación con el protocolo de Kioto, es decir, que a partir de ahora no firmen ni ratifiquen ningún tratado más de temática ambiental.

\section{EL IMPACTO AMBIENTAL DEL CRECIMIENTO ESTIMULADO POR EL COMERCIO "SIN INTERFERENCIAS".}

Lo que David Ricardo y el grueso de la tradición ortodoxa de la teoría económica nunca ha tenido en cuenta es el impacto ambiental de toda actividad productiva, lo que incluye el comercio mundial.

Desde la publicación del primer informe al Club de Roma, titulado Los límites del crecimiento, se ha convertido en un lugar común afirmar que no es posible un crecimiento de la producción que se hace para satisfacer las necesidades de una población que también crece de forma exponencial, y que exige un incremento acelerado del uso de recursos agotables y no agotables y de un incremento exponencial de la emisión de desechos y residuos. Lo cual no equivale a un mero crecimiento del

\footnotetext{
${ }^{16}$ Lo cual es, además, un síntoma claro de la pérdida de soberanía estatal.

17 Vid. SAN MARTÍN SÁNCHEZ DE MUNIÁIN, Laura: La OMC y la protección del Medio Ambiente. Aspectos jurídicos, Universidad Pública de Navarra, Pamplona, 2000, págs. 134 y ss. y bibliografía allí citada. Esta autora, por cierto, estima que es muy remota la posibilidad de que se produzca la citada contrarreforma. Haría bien, sin duda, en encomendarse a Santa Lucía y a sus poderes de conservación de la vista.

${ }^{18}$ Vid. GEORGE, S., op. cit., pág. 91.
} 
Producto Nacional Bruto (PNB), que es a lo que normalmente aluden economistas y ministros de economía cuando hablan de crecimiento.

Como ha explicado Michael Jacobs en La economía verde ${ }^{19}$, no hay que confundir el concepto de crecimiento del que se habla en el primer informe al Club de Roma, con crecimiento del PNB. Dado el carácter anacrónico de este concepto, que no refleja ni contabiliza el impacto ambiental, es perfectamente posible que un crecimiento del PNB vaya acompañado de un decrecimiento del impacto ambiental y también, como ha ocurrido en el África subsahariana durante la última década, que un decrecimiento del PNB vaya de la mano de un incremento de dicho impacto.

Si relacionamos lo anterior con la expansión del comercio mundial, entonces conviene señalar que, si éste aumenta, también se incrementará de entrada el transporte mundial de mercancías y, por ello, el uso de los combustibles fósiles. Esto, a su vez, generará un aumento de los niveles de contaminación. Recordemos que estamos hablando, por un lado, de recursos agotables y, por el otro, de una actividad que es causante de la emisión de más de la quinta parte de los gases responsables del efecto invernadero.

Las previsiones apuntan a que el transporte de personas y mercancías se va a multiplicar por tres durante los próximos 20 años, a un ritmo superior a un $6 \%$ anual. Con esa tasa se prevé que las reservas de petróleo de fácil acceso se agoten hacia el 2050 y que se produzca una aceleración en el aumento de la temperatura global $^{20}$.

Pero, más en general, como ha escrito Albert Recio: “ ... todo el proceso globalizador tiene un impacto ambiental negativo en la medida en que fomenta un proceso masivo de interrelación productiva a escala internacional, el cual genera la necesidad de construir grandes infraestructuras de comunicaciones y da lugar a un movimiento acelerado de bienes y personas sólo sostenible con un enorme derroche de materiales y energía." 21

Si tenemos en cuenta esta reflexión entonces podemos reconsiderar la supuesta "irreversibilidad" de dicho proceso. La mundialización productiva y financiera sólo podría ser "irreversible" en un mundo con una capacidad ilimitada para sostener la vida humana, lo que no es el caso. Negar esta evidencia es como negar -ahora sí- la ley de la gravedad.

En un planeta finito, no es posible alcanzar un desarrollo sostenible mediante un incremento de la producción que comporte un incremento exponencial del impacto sobre el medio ambiente. A partir de un determinado momento, ese tipo de crecimiento conduce a una situación de colapso ecológico que impide la satisfacción de las necesidades de la generaciones que lo padezcan y también, obviamente, de las generaciones futuras, lo que equivale a decir que impide alcanzar un desarrollo sostenible. En un mundo superpoblado, sumido en un caos climático, con una reducción brutal de la biodiversidad, sin grandes masas boscosas, con una delgada y agujereada capa de ozono, de recursos escasos o agotados, sin agua potable, plagado de toneladas

\footnotetext{
${ }^{19}$ JACOBS, Michael: La economía verde, Icaria/Fuhem, Barcelona, 1996., págs. 115 y ss.

${ }^{20}$ Vid. FERNÁNDEZ DURÁN, Ramón: “Transporte versus sostenibilidad” en El Ecologista, n 28 , pág. 49.

${ }^{21}$ RECIO, Albert: “ Multinacionales españolas” en El Ecologista, no 28, pág. 18.
} 
de residuos tóxicos y con un aire irrespirable, no son posibles ni la famosa globalización ni ninguna clase de progreso material para los seres humanos.

Los defensores de la mundialización neoliberal deben explicarnos todavía por qué el sistema depende tanto, si todo es tan irreversible, de la apropiación de recursos agotables como el petróleo, sin ir más lejos, y por qué los amos del mundo estiman que vale la pena gastar miles de millones de dólares en guerras como la del Golfo de 1991, la de Afganistán de 2001 o la de Irak pasado mañana ${ }^{22}$.

No está de más recordar que en 1991 se publicó una actualización del primer informe al Club de Roma, en el que se afirmaba que ya se habían sobrepasado algunos límites de sostenibilidad, en especial los que tenían que ver con el uso de algunos recursos y con la capacidad de los ecosistemas de absorber los desechos generados por la producción industrial. La conclusión del informe era clara: ya no se trataba sólo de impedir, como se proponía en 1972, el sobrepasamiento de los límites de sostenibilidad, sino que ahora se debía perseguir el objetivo de intentar "regresar desde más allá de los límites" 23 .

No se trata, pues, de favorecer en primer lugar el crecimiento económico y procurar después proteger el medio ambiente gracias a los medios proporcionados por lo primero, como de forma anacrónica se propone en el mencionado preámbulo del tratado de la OMC. Los agujeros en la capa de ozono, el caos climático, la brutal reducción de la biodiversidad o el aumento de la temperatura global deberían ser suficientes para convencer a todo el mundo de que el tipo de crecimiento impulsado hasta ahora ya es claramente insostenible y, por consiguiente, que de lo que se trata es de cambiar el modelo de desarrollo, no de hacer compatible el que hay con algunas medidas de protección del ambiente.

En este contexto, a lo único que nos puede conducir el crecimiento impulsado por el comercio es a que cada país intente atraer las inversiones de las empresas transnacionales prometiendo más explotación de los trabajadores y más devastación del medio ambiente que el vecino. Es lo que los críticos de la OMC llaman "la carrera hacia el abismo".

Lo más sensato es impulsar políticas económicas que favorezcan el desarrollo y al mismo tiempo el decrecimiento de su impacto ambiental. Eso con toda seguridad exige pensar en una dirección diferente y opuesta, en algunos casos, a los planteamientos teóricos de los que surgió la OMC y el proceso en curso de mundialización económica neoliberal ${ }^{24}$.

\footnotetext{
${ }^{22}$ Y la pérdida de cientos de miles de vidas humanas que provocan, se podría añadir; pero no está claro que eso les preocupe más que los dólares que cuestan.

${ }^{23}$ MEADOWS, Denis y Donella y RANDERS, Jorgen: Más allá de los límites del crecimiento, E1 País/Aguilar, Madrid, 1992.

${ }^{24}$ Algunas propuestas alternativas relativas al comercio las apunta Susan George en Pongamos la $O M C$ en su sitio, op. cit., págs. 95-102. Sobre las líneas generales de un modelo económico ecológicamente sostenible ver: BERMEJO, R. Economía sostenible, Bakeaz, Bilbao, 2002.
} 


\section{BIBLIOGRAFÍA}

Armborst, Stefan: "Liberalizar y comercializar todo bajo el sol" en El Ecologista, $\mathrm{n}^{\circ} 28$, especial "Globalización y medio ambiente", nov. 2001, pág. 9-13

Bermejo, Roberto: Libre comercio y equilibrio ecológico, Bakeaz, Bilbao, 1996.

Bermejo, Roberto: Economía sostenible., Bakeaz, Bilbao, 2001

Bourdieu, Pierre: Contrafuegos 2, Anagrama, Barcelona, 2001.

Commoner, Barry: En paz con el planeta, Crítica, Barcelona, 1992.

Fernández Durán, Ramón: "Transporte versus sosteniblidad" en El Ecologista, no 28 , págs. $48-52$.

George, Susan: Pongamos a la OMC en su sitio, Icaria, Barcelona, 2002.

Jacobs, Michael: La economía verde, Icaria/Fuhem, Barcelona, 1996.

Meadows, Denis y Donella; y Randers, Jorgen: Más allá de los límites del crecimiento, El País/Aguilar, Madrid, 1992.

Ost, François: “ La auto-organisation écologique des entreprises: un jeu sans conflits et sans règles?” en R.I.E.J., 1992/28, págs. 147-178.

Recio, Albert: “Multinacionales españolas” en El Ecologista, nº 28, págs. 17-19.

San Martín, Laura: La OMC y la protección del Medio Ambiente. Aspectos Jurídicos, Universidad Pública de Navarra, Pamplona, 2000.

UNCTAD: World Investment Report 2000, New York y Ginebra, 2001. 